\title{
Contribution of Peripheral and Central Fatigue in Different Conditions (Gender and Time of Day Differences)
}

\author{
by \\ Maryam Nourshahi ${ }^{1}$, Fatemeh Alirezaei², Farid Bahrpeyma ${ }^{3}$
}

\begin{abstract}
The aim of the present study was to examine the rate of central and peripheral contributions in circadian rhythms and gender differences after exhausted maximal exercise. From 36 volunteers, 12 healthy male and female subjects who had H-reflex (It is elicited by electrical stimulation and it has been suggested to be one of mechanisms that could cause central fatigue) were selected (mean age of $23.5 \pm 1.37$ years, height of $166.83 \pm 5.11 \mathrm{~cm}$, weight of $59.8 \pm 9.15 \mathrm{~kg}, V O_{2 \max }$ of $33.14 \pm 4.71 \mathrm{ml} . \mathrm{kg}^{-1}$. min-1in male subjects and mean age of $23 \pm 6.32$ years, height of $165.17 \pm 2.48 \mathrm{~cm}$, weight of $56 \pm 2.09 \mathrm{~kg}$ and $V O_{2 \max }$ of $29.05 \pm 2.89 \mathrm{ml} . \mathrm{kg}^{-1} \cdot \mathrm{min}^{-1}$ in female subjects) and took part in this investigation. Central (MVC, latency of H-reflex, amplitude of H-reflex and ratio of $H$-reflex to $M$-wave) and peripheral properties of fatigue (blood lactate and $M$-wave) were recorded before and after an exhausted maximal exercise at two different times of day: 9 am and $6 \mathrm{pm}$ which is separated by a week. Significant differences were found in HR, blood lactate and latency of $H$-reflex before and after exercise. However differences between male and female in MVC, amplitude of H-reflex, ratio of H-reflex to M-wave and M-wave was significant. With induced-exercise, central and peripheral fatigue was higher in men in the morning and in women in the afternoon., thus our results recommended designing suitable exercise programs for men and women in the afternoon and morning, respectively. However, further studies are needed to confirm these results, especially in recommending using transcranial magnetic stimulation.
\end{abstract}

Key words: central and peripheral fatigue, circadian rhythms, gender differences

\section{Introduction}

Fatigue is a complex process that is defined as a reduction in force generating capacity, or the inability to maintain performance (Fulco, 1999). The development of this temporary loss of force is a complicated phenomenon which may develop due to dysfunction of any critical cites on the pathways from the central nervous to the peripheral sys-
tems(Gandevia, 2001), such as motor unit recruitment, firing rate and chemical transmission across neuromuscular junctions (Bigland-Ritchie, 1984), propagation of action potential along the muscle membrane and $\mathrm{T}$ tubules, $\mathrm{Ca}^{2+}$ release from sarcoplasmic retinaculum, $\mathrm{Ca}^{2+}$ binding to troponin $\mathrm{C}$ and cross-bridge cycling (Mclester, 1997).

In analyzing the site of force origination, causes of fatigue can be divided into peripheral and central factors, whereby neuromuscular junctions and mus-

1 - Department of Exercise Physiology, Faculty of physical Education and sport Sciences, Shahid Beheshti University G.C, Tehran, Iran

2 - Department of Sport Physiology, Tarbiat moallem University, Tehran, Iran

3 - Department of Physiotherapy, Tarbiat modares University, Tehran, Iran 
cle tissue are regarded as peripheral sites and the higher systems as the central sites (Gandevia, 2001).

Different studies have used EMG as a fatigue index. The strength of the excitation achieved by a same sized compound Ia afferent action potential on its motorneuron pool can be measured by means of Hoffman-reflex (Kent-Braun, 1999). A decrease in the H-reflex can be attributed to decreased motorneuron excitability or increased pre-synaptic inhibition of Ia afferent neuron (Hugon, 1973). Decreased excitation from Ia afferent neuron due to decreased firing frequencies of muscle spindles has been suggested to be one of mechanisms that could cause central fatigue (Nourdland, 2003; Macefield, 1999).

Several processes are involved in converting an axonal action potential in to a sarcoma action potential. Collectively, these processes are referred as neuromuscular propagation. Sustained activity can impair some of the processes involved in neuromuscular propagation, and this can contribute to the decline in force associated with fatigue. The most common way to test impairment of neuromuscular propagation is to elicit M-waves before, during and after a fatiguing contraction (Sandiford, 2004). Mwave is measured by applying an electric shock to a nerve to generate action potential in the axons of alpha motorneurons and measuring the EMG response in muscle (Enoka, 1994; Fuglevand, 1993).

There are experimental evidences to show that the decrease in intracellular $\mathrm{pH}$, related to the increase in $\mathrm{H}^{+}$concentration associated with lactate production could well explain the force reduction, observed during fatigue (Stackhouse, 2001). Although fatigue has both central and peripheral components, but whose relative contribution to fatigue may be task dependent. Fatigue ability is various between muscle groups (Behm, 2002), muscle action types (Löscher, 2002) and individuals (Westing, 1999). In addition to variations in the extent and mechanism of fatigue with differences in task performance, some studies have suggested that fatigue may be influenced by gender (Hunter, 2001) and times of day (Castaingts, 2004). The majority of these studies have shown that women exhibit greater resistance to fatigue than men during maximal isometric contractions. Moreover, the results of other studies have suggested that the greater fatigue resistance of women declines as the intensity of the contraction increases (Maughan, 1986; Kent-Braun, 2002). Hunter\& Enoka (2001) pointed to a disappearance in related fatigue differences between men and women.
In adult women, circulating estrogen and progesterone levels vary normally throughout the menstrual cycle that can cause variations in cardiovascular, respiratory and metabolic parameters (Constantini, 2005). Nevertheless different times of day may affect women gender steroids with subsequent implications for performance.

Circadian rhythms are internally generated phenomena with periodicity of 24 hours (Aschoff, 1995). Many factors could affect internal body environment, such as core body temperature and hormones result in the body experiences various conditions which can influence on exercise in different times of day (Guette, 2005a). Guette et al (2005b) showed that the quadriceps muscle torque changes in a predictable manner during a $24 \mathrm{~h}$ period--changes, which are linked to muscular rather than neural level. Castigaignts et al (2004) reported weaker neuromuscular function in the evening that this weaker performance of triceps sura muscle was related to a higher fatigue in activated motor units (Castigaignts, 2004). Nevertheless it is less known about the effect of times of day on fatigue.

Since no studies have been conducted to investigate fatigability differences between men and women, in different times of day and the importance of these parameters in a train programming, Therefore, our aim was to determine the rate of contribution of central (H-reflex, MVC) and peripheral (Mwave, lactate) fatigue, following exhaustive maximal exercise in various condition (gender and times of day). It was hypothesized that men and women responded differently in the morning and evening to the maximal exhausting exercise.

\section{Methods}

Subjects: Thirty-six healthy physical education students from Tehran universities, with at least a 2 year history of physical activity, were volunteered. Six men and six women of whom had H-reflex, were chosen as research subjects (mean age of $23.5 \pm 1.37$ years, height of $166.83 \pm 5.11 \mathrm{~cm}$, weight of $59.8 \pm 9.15$ $\mathrm{kg}, \mathrm{VO}_{2 \max }$ of $33.14 \pm 4.71 \mathrm{ml} . \mathrm{kg}^{-1}$. min-1in male subjects and mean age of $23 \pm 6.32$ years, height of $165.17 \pm 2.48 \mathrm{~cm}$, weight of $56 \pm 2.09 \mathrm{~kg}$ and $\mathrm{VO}_{2 \max }$ of $29.05 \pm 2.89 \mathrm{ml} \mathrm{kg}^{-1}$. min-1 in female subjects).

Procedure: A couple days before experimental session, subjects familiarized with proper nutrition guidelines, activity and proper exercise clothing during testing .In the experimental session, all subjects completed health questionnaire and general in- 
formation form, and written informed consent was obtained. Each subject's body mass, height and resting heart rate using belt was determined. Following 10 min rest, blood samples were drown from a finger to measure resting blood lactate concentration using lactometer(CE483, Germany). Subjects then layed prone to obtain an H-reflex. MVC of plantar flexors were measured 3 times, with 30s rest between each trial. The subjects then began the fatiguing protocol (Storer \& Davis, 1998) on an ergometer (Monark, Taiwan). The exercise protocol consisted of maximal training until exhaustion. After exhaustion, power and heart rate were registered. Immediately after fatiguing protocol, lactate and MVC were recorded, and H-reflex was performed. All subjects completed tests 2 times: 9 am and $6 \mathrm{pm}$, within a time interval of 4 to 7 days. Women were tested in the same menstrual cycle phase.

H-reflex recording: The H-reflex was elicited by electrical stimulation of the peripheral or tibial nerve in the political fossa of the soleus muscle (Simonsen, 1999). For this reason, subjects lay prone, with the cathode electrode placed on the tibial nerve and proximally, relative to anode electrode. The stimulus consisted of $1 \mathrm{~ms}$, such that no further Ia afferent neuron could be stimulated (Burke, 1989). The optimum site to elicit the H-reflex was marked. The stimulus intensity increased gradually until H-reflex and M-wave were obtained.

Fatiguing protocol: After adjusting the seat of cycle ergometer with the heel on the pedal and leg straight (Adams \&Beam 2008), the subjects performed an incremental exercise test with a $15 \mathrm{~W} / \mathrm{min}$ increment until volitional fatigue, after a 4- min warm up with no load. The maximal watts attained during the exercise are used in gender-specific equations that also involve body weight and age for calculating $\mathrm{VO}_{2} \max$ (Equation 1,2).

\section{Men: $\mathrm{VO}_{2} \max (\mathrm{ml} \cdot \min )=[10.51(\mathrm{~W}, \max )]+[6.35(\mathrm{wt}, \mathrm{kg})]-$

$$
\text { [10.49(age,yr)]+519.3 (Eq.1) }
$$$$
\text { Women: } \mathrm{VO}_{2} \max (\mathrm{ml} . \mathrm{min})=
$$ \\ [9.39(W,max $)]+[7.7(w t, k g)]-[5.88($ age,yr $)]+136.7$ \\ (Robergs\&Keteyian 2003).}

Statistical analysis: The $\mathrm{K}-\mathrm{S}$ was used to test the normality of data. Descriptive statistics was used to present data as means \pm SD. To test for changes in the dependent variables before and after fatigue protocol, repeated measure ANOVAs (gender as between group factor, time and fatigue as within group factors) were preformed for each variable. In the event of a significant main effect, Bonfferoni test was used. All statistical significance was established at $\mathrm{p} \leq 0.05$.

\section{Results}

Maximal exercise and gender had significant effect on heart rate (respectively $\mathrm{F}_{1,10}=697.7, \mathrm{p}=0.000$; $\mathrm{F}_{1,10}=14.8, \mathrm{p}=0.003$ ). Time of day had no significant effect on HR. HR response was greater in the morning in women and in the evening in men.

Lactate concentration increased significantly following maximal exercise $\left(\mathrm{F}_{1,10}=0.81, \mathrm{p}=0.000\right)$ (Figure 1). The data was significantly different in genders after maximal exercise $\left(F_{1,10}=10.85\right.$, $\left.\mathrm{p}=0.008\right)($ Figure 1).

We observed a significant effect of maximal exercise on delaying $\mathrm{T}$ (the time between stimulation and response of muscle). In addition, the effect of sex on H-reflex amplitude was detected .Men tended to exhibit a greater decline in H-reflex amplitude than women in the morning, while women showed greater decline in the evening (Figure 2).

No significant changes were found in M-wave after maximal exercise $\left(F_{1}, 10=1.62, p=0.023\right)$. Meanwhile, time of day and sex had significant effect on M-wave (respectively, $F_{1}, 10=6.06, p=0.034$ and $F_{1}$,

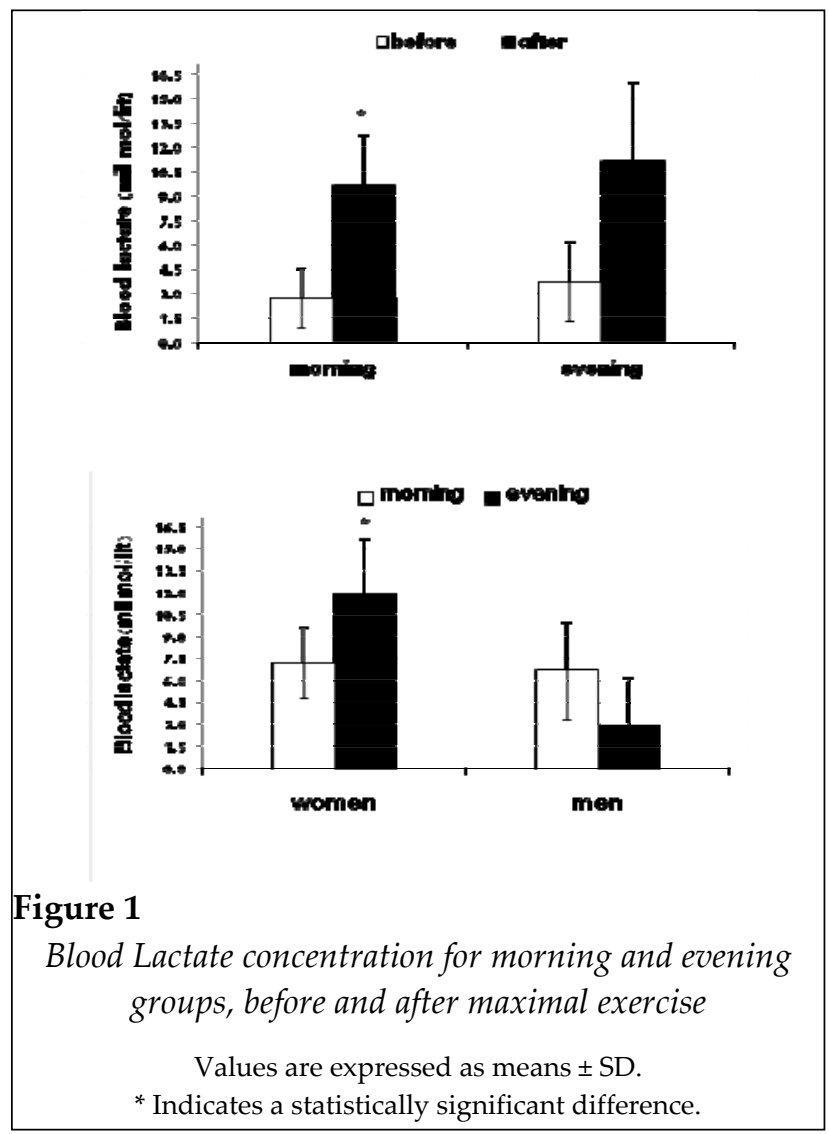




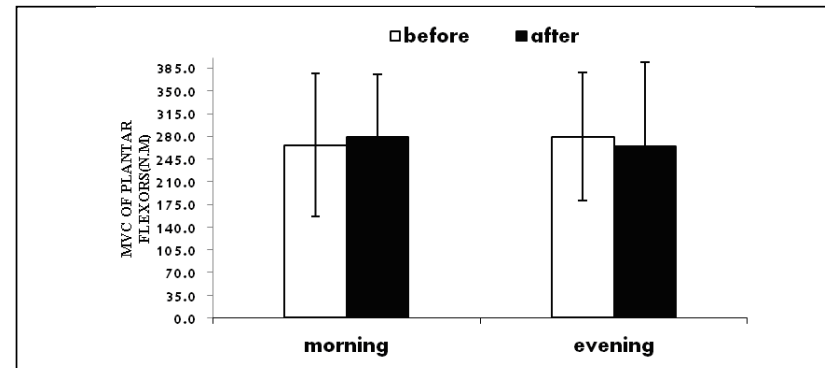

Figure 2

Maximum voluntary contraction $(M V C)$ of plantarflexors for morning and evening groups, before and after maximal exercise
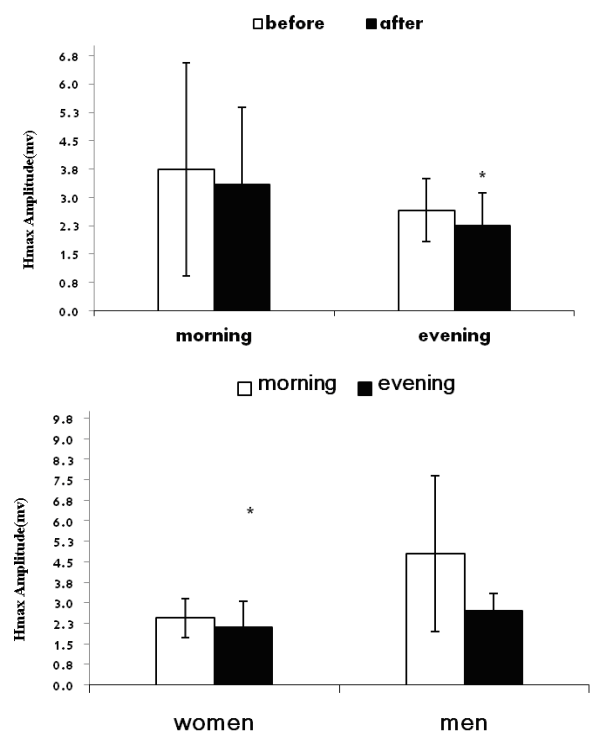

Figure 3

Maximal amplitude of $\mathrm{H}$ reflex ( $\mathrm{H}$ max amplitude) for morning and evening groups, before and after maximal exercise

Values are expressed as means \pm SD

* Indicates a statistically significant difference.

$10=8.98, \mathrm{p}=0.013$ ). These declines were greater in the morning for men and in the evening for women (Figure 3). Gender had significant effect on strength of plantar flexors after maximal exercise. $\left(\mathrm{F}_{1}, 10=\right.$ 10.85, $\mathrm{p}=0.008$ ) (Figure 4). Hmax/Mmax ratio had no significant change following maximal exercise and between genders in different times of day.

\section{Discussion}

Results demonstrated that heart rate increases after maximal exercise in the morning and evening. HR response was significantly different between men and women after exercise. These results are in agreement with some studies (Ryon, 1994; Davis, 2000; O'Toole, 1989) but not with others (Melanson,

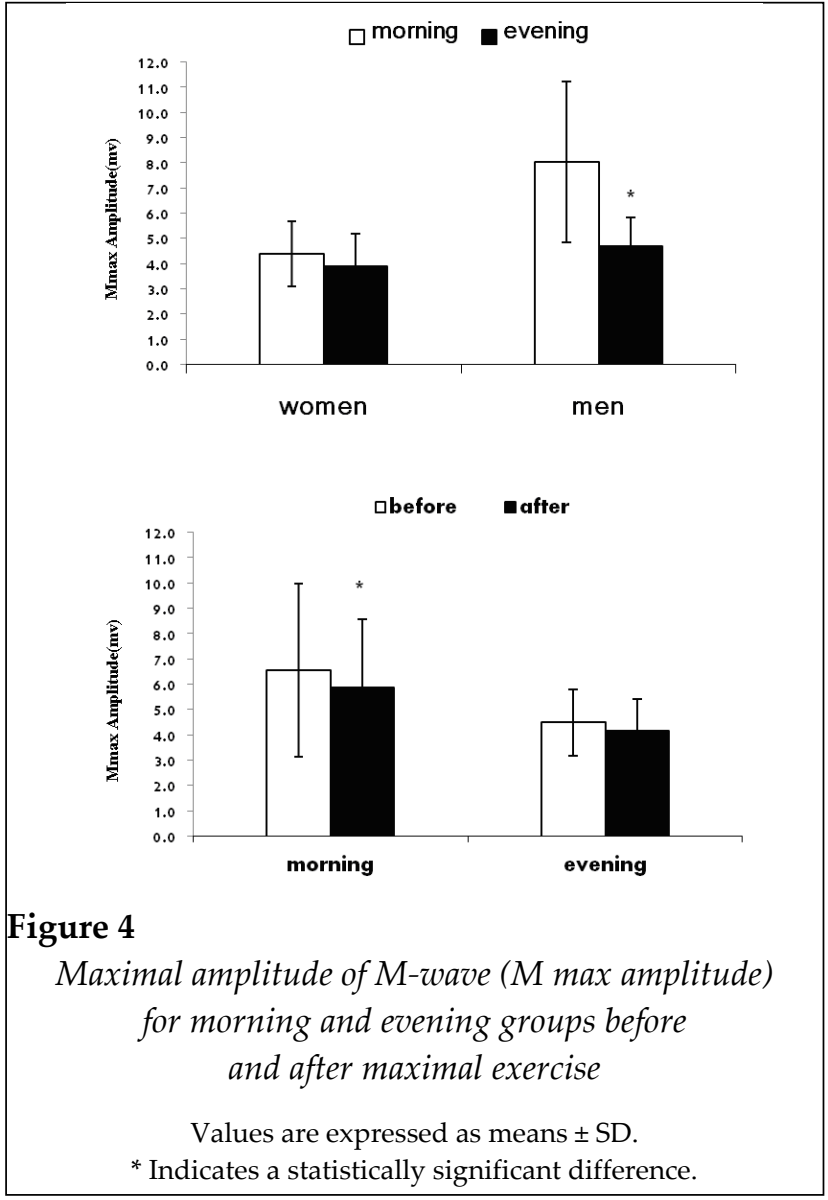

2002). It is clear that women and men show different physiologic responses to exercise. Of the different responses, sinus heart rhythm is a function of sex. Ryan et al. (1994) showed that high frequency power spectrum of heart rate and parasympathetic function is greater in women (Hargreaves, 1998). Moreover, differences in utilization of energy supplies (Darleen, 2002; Russ, 2003), energy metabolism pathways (KentBraun, 2002), vascular diffusion (Hatzikotoulas, 2004) and rate of recruitment of motor units (Melanson, 2002) can be contributed to these varieties.

Differences in metabolic responses between men and women show more reliance of men to non-oxidative ATP sources (Hicks, 2001). The greater oxidative capacity of women allows them to utilize the available oxygen in a more efficient manner than men; while a greater rate of glycolysis in men would be associated with greater rates of carbohydrate oxidation (Kent-Braun, 2002).

The present findings point to significant changes after maximal exercise, and sex factor differences showed a significant effect on lactate changes, with greater changes occurring in men during the morning and evening. These results were in agreement with Russ \& Kent-Braun (2003), which demonstrated 
a correlation between lactate accumulation and fatigue, where $\mathrm{H}^{+}$is a by-product of glycolysis and thereby involved in fatigue (Russ, 2002). The buildup of $\mathrm{H}^{+}$within the muscle lowers the blood $\mathrm{Ph}$ and may reduce muscle force by decreasing $\mathrm{Ca}^{2+}$ from the sarcoplasmic retinaculum, thereby decreasing the sensivity of troponin $\mathrm{C}$ to $\mathrm{Ca}^{2+}$ and interfering with cross-bridge cycling (Mclester, 1997).

Differences in metabolic responses between men and women show more reliance of men to non-oxidative ATP sources (Hicks, 2001). The greater oxidative capacity of women allows them to utilize the available oxygen in a more efficient manner than men; while a greater rate of glycolysis in men would be associated with greater rates of carbohydrate oxidation (Kent-Braun, 2002). In addition, differences in muscle mass and neuromuscular activation patterns may explain gender differences in fatigability. Although all women tests were taken in the same menstrual phase, but probably some of the gender differences were related to the influence of menstrual cycle. Even though most researches suggests that oxygen consumption, heart rate and rating of perceived exertion responses to sub- maximal exercise are not affected by the menstrual cycle; several studies report a higher cardiovascular strain during moderate exercise in the mid-luteal phase. Nevertheless, time to exhaustion at maximal exercise intensities showed no change over the menstrual cycle (Constantini, 2005).

No significant differences were observed in strength of plantar flexors after maximal exercise. Changes in strength are dependent on various factors, such as exercise protocol and specific muscle involvement (Garland, 2002). Since the soleus muscle is more oxidative than other muscles during plantar flexion (Gardiner, 2001), despite soleus muscle fatigue, there were no changes observed in MVC (ACSM, 2006). Sometimes fatigue occurs without any change in force (Gardiner, 2001). Moreover, plantar flexors are more resistant to fatigue than dorsiflexors (Gardiner, 2001).

In this experiment, it was shown that time of day had no effect on muscle strength following maximal exercise. Contrary, Castigaignts et al. (2004) observed less neuromuscular efficiency and Gutte et al. (2005a) reported a decline in torque force of the plantar flexors in the evening. Castigaignts et al. (2004) argued that most fatigable motor units could be affected by daily activity. Martin et al. (1989) advanced the hypothesis that Pi concentration and calcium movements in muscle fibers could explain the diurnal variation of strength. In contrast with the diurnal variation observed in concentric contractions, in which some researches relate these variations to the metabolic and ionic factors involved in circadian rhythm, it was found that time of day had no effect upon eccentric tasks (De Ruitier, 2001). However, more investigations are needed to find the effect of time of day on strength.

Gender had significant effect on decline in plantar flexor strength following sub-maximal training. These findings are in agreement with Fulco et al. (1999), Garland \& Gossen (1991) and Hicks et al. (2001), who believe that sex differences, may affect fatigability. But in Hatzikotoulas et al. (2004) findings, men and women were matched for strength, and gender differences were not observed. Furthermore, differences between men and women can be attributed to non-matched subjects for strength. Hreflex amplitude showed decrease after maximal exercise but was not statistically significant. This decrease in the H-reflex amplitude can be attributed to decreased motorneuron excitability or increased presynaptic inhibition of Ia afferents (Chen, 2002).

The present study demonstrated no significant change in M-wave amplitude following maximal exercise, but the change was significant between men and women at different times of the day. Women had experienced more declines in the morning and men had shown more declines in the evening. Moreover, peripheral fatigue, which is attributed to neuromuscular fatigue and intracellular metabolism, was observed more in women rather than in men. These results were in agreement with Tanino et al. (2005). A decline in M-wave amplitude is interpreted as an impairment of one or more of the processes involved in converting the axonal action potential in to a muscle action potential (Enoka, 1994).

Hmax/Mmax ratio had no significant change following maximal exercise or between genders at different times of day. To our knowledge no research has investigated the relationships between men and women in the morning and evening after maximal exercise. Guette et al. (2005) observed no change in Hmax/Mmax ratio as an index of efficiency in transition of reflex between Ia afferents and motorneuron pool, at different times of day. Castigaignts et al. (2004) reported similar findings, suggesting a lack of diurnal fluctuation in the proportion of motor units reflexively activated. Thus, it is postulated that since the Hmax/Mmax ratio did not change in the present 
study, it seems that the reflex pathway was not affected by daily activity at rest.

Hmax/Mmax ratio had no significant change following maximal exercise or between genders at different times of day. To our knowledge no research has investigated the relationships between men and women in the morning and evening after maximal exercise. Guette et al. (2005) observed no change in Hmax/Mmax ratio as an index of efficiency in transition of reflex between Ia afferents and motorneuron pool, at different times of day. Castigaignts et al. (2004) reported similar findings, suggesting a lack of diurnal fluctuation in the proportion of motor units reflexively activated. Thus, it seems that the reflex pathway was not affected by daily activity at rest.

\section{Conclusion}

Fatigue has been the subject of many investigations, but the important fact obtained from the results is that measured peripheral fatigue indices (Mwave amplitude and lactate) in women were greater than in men. Men had a greater decrease in the morning and women had bigger decline in the evening in peripheral fatigue factors. These changes were unexpected because reduced vascular occlusion occurred during muscle contraction as a result of lower absolute forces; thus more vascular diffusion and less ischemic condition is expected during contraction. Subsequently, women have more dependency on oxidative capacity. But from the reflective pathways of central fatigue indices (H-reflex, time latency of H-reflex, MVC, Hmax/Mmax ratio), after maximal exercise, the major results of this study revealed a difference in central fatigue between genders. The H-reflex was greater in the morning for men and greater in the evening for women.

With induced-exercise, central and peripheral fatigue was higher in men in the morning and in women during the evening. Based on our results, we recommend designing suitable training programs for men and women in the afternoon and morning, respectively. However, more research is needed to confirm these result, especially when recommending the use of transcranial magnetic stimulation.

\section{References}

Adams GM and WC Beam. Exercise physiology Laboratory Manual. Fifth edition. Mc Grow Hill:141 2008.

American college of sports medicine. ACSM's Advanced Exercise Physiology. lippincott Williams\&Wilkins 2006.

Aschoff J. Circadian rhythms in man. Science, 1995. 148:1427-1432.

Behm DG, Whittle J, Button D, and Power K. Inter muscle differences in activation. Muscle Nerve 2002. 25: 236243.

Bigland-Ritchie B, Woods JJ. Changes in muscle contractile properties and neural during human muscular fatigue. Muscle Nerve. 1984. 7:691-699.

Burke D, Adams RW, and Skuse NF. The effect of voluntary contraction on the H-reflex of human limb muscles. Brain. 1989. 112:417-433.

Castaingts V, Martin A, Van Hoecke J, Perot C. Neuromuscular efficiency of the triceps surae in induced and voluntary contractions: morning and evening evaluations. Chronobiol Int, 2004. 21:515-527.

Constantini NW, Dubnov G, Constanse ML. The menstrual cycle and sport performance. Clin Sports Med. 2005. 24:51-82.

Chen XY, Chen L, Wolpaw JR and Jakman LB. Corticospinal tract transaction reduces H-reflex circadian rhythm in rats.Brain research, 2002. 942(1-2): p101-108.

Darleen A, Sandoval S, Katheleen S. Gender differences in the endocrine and metabolic responses to hypoxic exercise. Appl Physiol 2002. 92: 504-512.

Davis, SN, Galassett P, Wasserman DH, and Tate D. Effects of gender on neuroendocrine and metabolic counter regulatory responses to exercise in normal man. J Clin Endocrinol Metab 2000. 85: 224-230.

De Ruitier CJ and De Haan A. Similar effects of cooling and fatigue on eccentric and concentric force-velocity relationships in human muscle, J Appl Physiol 2001.90: 2109-2116. 
Enoka RM. Neuromechanical Basis of Kinesiology. Human kinetics. Second edition. 1994. P 283-288. P: 177-179.

Fulco CS, Rock PB, Muza SR, Lammi E, Cymerman A, Butterfield G, Moore LG, Braun B, Lewis SF. Slower fatigue and faster recovery of the adductor policis muscle in women matched for strength with men. Acta Physiol Scand 1999.167:233-239.

Gandevia SC. Spinal and super spinal factors in human muscle fatigue. Physiol Rev. 2001. 81:1725-1789.

Gardiner FPH. Neuromuscular aspects of physical activity. Human Kinetics. 2001.

Garland SJ, Gossen. E.R. The muscular wisdom hypothesis in human Muscle fatigue Exerc Sport sci Rev. 2002.30(1): 45-49.

Guette M, Gondin J, Martin A. Morning to evening changes in the electrical and mechanical properties of human soleus motor units activated by H-reflex and M-wave. Eur J Appl Physiol, 2005a. 95:377-381.

Guette M, Gondin J, Martin A. Time -of- day effect on the torque and neuromuscular properties of dominant and non-dominant quadriceps femoris. Chronobiol Int, 2005b. 22:1-18.

Hargreaves M, Mckenna MJ, Jenkins DG, Warnington SA, Li JL, Snow RJ, and Febbraio MA. Muscle Metabolites and performance during high-intensity, intermittent exercise. J. Appl. Physiol. 1998. 1687-1691.

Hatzikotoulas K, Siatras T, Spyropouiou E, Paraschos I, Patikas D. Muscle fatigue and elecromyographic changes aren't different in women and men matched for strength. Eur J Appl Physiol, 2004. 92:298-304.

Hicks Al, Kent-Braun JA, and Ditor DS. Gender differences in human skeletal muscle fatigue. Exerc. Sport. Sci. Rov. 2001. 29: 109-112.

Hunter KS, Enoka RM. Gender differences in the fatigability of arm muscles depend on absolute force during isometric contractions. J Appl physiol. 2001. 91: 2686-2694.

Hunter SK, Duchateau J, Enoka RM. Muscle fatigue and the mechanisms of task failure. Exerc Sport Sci Rev. 2004. 32(2): 44-49.

Kent-Brown JA, Ng AV, Doyle JW, and Towse TF. Human skeletal muscle responses vary with age and gender during fatigue due to incremental exercise. J. Appl. Physiol. 2002. 93:1873-1823.

Kent-Brown JA. Central and peripheral contributions to muscle fatigue in humans during sustained maximal effort. Eur J Appl Physiol. 1999. 80:57-63.

Löscher WN and Nordlund MM. Central fatigue and motor cortical excitability during repeated shortening and lengthening actions. Muscle Nerve 2002.25: 864-872.

Macefield G, Hagbarth KE, Gorman R, Gandevia SC, and Burke D. Decline in spindle support to alphamotoneurones during sustained voluntary contractions. J Physiol 1999. 440: 497-512.

Marino FE. Anticipatory regulation and avoidance of catastrophe during exercise - induced hyperthermia. Comp Biochem Physio B Biochem Mol Biol. 2004. 139: 561-569.

Martin A, Carpentier A, Guissard N, Van Hoecke A. Effects of ATP, ADP and Pi. J Musc Res Cell Motil: 1989. 135146.

Maughan RJ, Harman M, Leiper JB, Sale D and Delman A. Endurance capacity of untrained males and females in isometric and dynamic muscular contractions. Eur. J. Appl. Physiol. 1986. 55:395-400

Mclester JR. Muscle contraction and fatigue: the role of adenosine de phosphate and inorganic phosphate. Sport Med. 1997. 23:287-305.

Melanson EL, Sharp TA, Seagle HM, Horton TJ, Dunahoo WT, Grunwald GK, Hamilton TJ, Hill JO. Effect of exercise intensity on 24-h energy expenditure and nutrient oxidation. J Appl physiol. 2002. 92: 1045-1052.

Nordlund MM, et al. Central and peripheral contributions to fatigue in relation to level of activation during repeated maximal voluntary isometric plantar flexions. J. Appl. Physiol 2003. (96): 218-225. 
O'Toole LM. Gender differences in cardiovascular responses to exercise. Cardiovasc Clin, 1989. 19(3):17-33.

Robergs RA and SJ Keteyian. Exercise physiology. Second edition. McGraw Hill: 2003. 297

Russ D, Kent-brown J. Gender differences in human skeletal muscle fatigue are eliminated under ischemic conditions. J Appl Physiol, 2003. 96(9):2414-2442.

Ryan SM, Goldberger AL, Pincus SM, Mietus J, Lipsitz LA. Gender and age related differences in heart rate dynamics: are women more complex than men? J Am Coll Cardiol, 1994. 24(7):1700-7.

Sandiford SD, Green TA, Duhamel JG, Perco JD, Ouyang, J. Inactivation of human muscle Na- K- ATPase in vitro during prolonged exercise is increased with hypoxia. J Appl physiol. 2004. 96: 1764-1775.

Simonsen EB and Dyhre PP. Amplitude of the human soleus H-reflex during walking and running. J Physiol. 1999. 515:929-939.

Stackhouse SK, Reisman DS, Binder-Macleod SA. Challenging the role of ph in skeletal muscle fatigue. Physical Therapy. 2001. 1897-1902.

Tanino Y, Daikuya S, Nishimori T, Takasaki K, Suzuki T. M-wave and H-Reflex of soleus muscle before and after electrical simulation in healthy subjects. J Electromyogr Clin Nerophysiol, 2003. 43(6):381-4.

Westing SH, Cresswell AG, and Thorstensson A. Muscle activation during maximal voluntary eccentric and concentric knee extension. Eur J Appl Physiol 1999. 62: 104-108.

\section{Corresponding author}

\section{Maryam Nourshahi}

Department of Exercise Physiology, Faculty of Physical Education and Sport Sciences, Shahid Beheshti University, G.C,.Evin 198396113, Tehran, Iran

phone: +982129902942

fax: +98 2122431953

e-mail: m-nourshahi@sbu.ac.ir 